\title{
Strain effects in group-III nitrides: deformation potentials for AlN, GaN, and InN
}

\author{
Qimin Yan ${ }^{1}$, Patrick Rinke ${ }^{1}$, Matthias Scheffler ${ }^{1,2}$, and Chris G. Van de Walle ${ }^{1}$ \\ ${ }^{1}$ Materials Department, University of California, Santa Barbara, CA 93106-5050, USA \\ ${ }^{2}$ Fritz-Haber-Institut der Max-Planck-Gesellschaft, Faradayweg 4-6, D-14195 Berlin, Germany
}

(Dated: May 31, 2010)

\begin{abstract}
A systematic density functional theory (DFT) study of strain effects on the electronic band structure of the group-III nitrides (AIN, GaN and InN) is presented. To overcome the deficiencies of the local-density and generalized gradient approximations (LDA and GGA) the Heyd-Scuseria-Ernzerhof hybrid functional (HSE) is used. Cross-checks for GaN demonstrate good agreement between HSE and exact-exchange based $G_{0} W_{0}$ calculations. We observe a pronounced nonlinear dependence of band-energy differences on strain. For realistic strain conditions in the linear regime around the experimental equilibrium volume a consistent and complete set of deformation potentials is derived.
\end{abstract}

PACS numbers: $71.20 . \mathrm{Nr}, 71.70 . \mathrm{Fk}, 85.60 . \mathrm{Bt}$

The group-III nitride compounds and their alloys have recently received considerable attention as a versatile materials class. AlN, GaN and $\mathrm{InN}$ all crystallize in the wurtzite structure, but have vastly different band gaps ranging from $6.0 \mathrm{eV}$ for $\mathrm{AlN}$ down to $0.7 \mathrm{eV}$ for InN. For light emitting diodes (LEDs) ${ }^{1}$ and laser diodes (LDs) $)^{2,3}$ the group-III-nitrides are currently the only commercially available materials class for the green to the deep ultraviolet part of the spectrum, and future applications as chemical sensors, ${ }^{4}$ in quantum cryptography ${ }^{5}$ or in photocatalysis ${ }^{6}$ are being explored. Applications in solid state lighting, however, are currently limited by loss mechanisms ${ }^{7,8}$ and a deeper understanding of the fundamental materials properties is required. One crucial factor is the effect of strain.

Due to the large differences in lattice parameters and thermal expansion coefficients between the substrate and the nitride overlayers, and between nitride layers with different alloy compositions, strain is always present in group-III-nitride based devices. Strain influences the optical properties ${ }^{9-11}$ in particular the energy of optical transitions, and for nonpolar or semipolar devices the polarization of the emitted light. ${ }^{12}$ The effects of strain are characterized by the change of a transition energy (energy difference) upon application of strain, and the linear coefficient is defined as deformation potential. Together with the Luttinger (band) parameters, the deformation potentials constitute essential input for device modeling. ${ }^{13}$

The experimental determination of deformation potentials is quite difficult, and aggravated by the fact that not all strain components can be determined accurately or without further approximations and that the deformation potentials cannot be isolated from each other, because uniaxial and biaxial strain cannot be applied separately. As a result the experimental data for GaN are scattered over a very large range. ${ }^{10,11,14-17}$ For AlN and InN no experimental data are available, except for the hydrostatic deformation potential of the band gap in InN. ${ }^{18}$

Previous theoretical studies have also produced widely differing values, resulting in a large uncertainty range. ${ }^{19-21}$ The error bars can, in part, be attributed to the band-gap problem of density functional theory (DFT) in the local-density or generalized gradient approximations (LDA and GGA). Moreover, not all previous studies include a relaxation of the internal displacement parameter $u$, which sensitively affects the crystal-field split-
TABLE I: Equilibrium lattice parameters $(a$ and $c$ ) and band gaps $\left(E_{g}\right)$ obtained with LDA, GGA, and HSE. Experimental lattice parameters at $T=300 \mathrm{~K}$ and band gap values are taken from Refs. 26 and 13.

\begin{tabular}{cccccc}
\hline \hline & Method & $\mathrm{a}(\AA)$ & $\mathrm{c}(\AA)$ & $\mathrm{u}$ & $E_{g}(\mathrm{eV})$ \\
\hline AlN & LDA & 3.092 & 4.950 & 0.3818 & 4.40 \\
& GGA & 3.127 & 5.021 & 0.3812 & 4.10 \\
& HSE & 3.102 & 4.971 & 0.3819 & 5.64 \\
& Exp. & 3.112 & 4.982 & - & 6.25 \\
\hline GaN & LDA & 3.155 & 5.145 & 0.3764 & 2.12 \\
& GGA & 3.215 & 5.240 & 0.3766 & 1.74 \\
& HSE & 3.182 & 5.173 & 0.3772 & 3.24 \\
& Exp. & 3.190 & 5.189 & - & 3.51 \\
\hline InN & LDA & 3.504 & 5.670 & 0.3784 & $<0$ \\
& GGA & 3.573 & 5.762 & 0.3792 & $<0$ \\
& HSE & 3.548 & 5.751 & 0.3796 & 0.68 \\
& Exp. & 3.540 & 5.706 & - & 0.78 \\
\hline \hline
\end{tabular}

ting. The equilibrium lattice parameters around which the linear expansion is performed also play a role, as we will demonstrate, due to pronounced nonlinearities. In this work we use the Heyd-Scuseria-Ernzerhof (HSE) ${ }^{22,23}$ DFT hybrid functional, which produces band gaps and equilibrium lattice parameters in much better agreement with experiment than LDA and GGA.

We present a complete set of deformation potentials $\left(a_{c z}-D_{1}, a_{c t}-D_{2}, D_{3}, D_{4}\right.$, and $\left.D_{5}\right)$ for the wurtzite phases of AlN, GaN and InN. Our first-principles calculations are performed using the plane-wave projector augmented wave (PAW) and hybrid functional method as implemented in the VASP code. ${ }^{24}$ The screening parameter $\mu$ in HSE was fixed at a value of 0.2 (HSE06) and the mixing parameter $\alpha$ was set as 0.25 . LDA and GGA-PBE ${ }^{25}$ calculations are performed for comparison. The semi-core $d$-electrons of $\mathrm{Ga}$ and $\mathrm{In}$ are treated as valence electrons. We use $6 \times 6 \times 4 \Gamma$-point centered $k$-point mesh and a plane-wave cutoff of $600 \mathrm{eV}$. Such a large plane-wave cutoff was found necessary to determine the internal displacement parameter $u$ accurately, which sensitively affects the valence band structure, most notably the crystal-field splitting.

Table I demonstrates that LDA underestimates and GGA overestimates the equilibrium lattice parameters of AlN, GaN and InN, while HSE provides the best agree- 
ment with the experimental values. Band gaps are underestimated severely in LDA and GGA, which for InN even results in a negative gap (metallic state). HSE again shows a considerable improvement and produces gaps that are close to those obtained from exact-exchange based $G_{0} W_{0}$ calculations (to within $0.1 \mathrm{eV}$ for $\mathrm{GaN}$ and $\mathrm{InN})^{26}$ and only slightly underestimate the experimental values.

The strain-induced band-structure modifications in the vicinity of the $\Gamma$-point have been derived from the $\mathbf{k} \cdot \mathbf{p}$ approach by Bir and Pikus. ${ }^{27}$ Solving the unstrained $6 \times 6$ Hamiltonian for the valence bands yields three eigenstates corresponding to the heavy hole $(\mathrm{HH})$, light hole $(\mathrm{LH})$, and crystal-field split-off band $(\mathrm{CH})$. The transition energies to the conduction band are denoted as $E_{A}$, $E_{B}$, and $E_{C}$, respectively. Here we do not consider the spin-orbit interaction $\left(\Delta_{2}=\Delta_{3}=0\right)$ so that for the unstrained system the $\mathrm{HH}$ and $\mathrm{LH}$ bands become doubly degenerate $\left(\Gamma_{6}\right)$ and the $\mathrm{CH}$ band $\left(\Gamma_{1}\right)$ is split off by the crystal-field splitting. For the special case that $\varepsilon_{x x}=\varepsilon_{y y}$ (which preserves the symmetry in the $c$ plane), adding strain to the Hamiltonian changes the transition energies in the following way:

$$
\begin{aligned}
E_{A / B}= & E_{A / B}(0)+\left(a_{c z}-D_{1}\right) \varepsilon_{z z}+\left(a_{c t}-D_{2}\right) \varepsilon_{\perp} \\
& -\left(D_{3} \varepsilon_{z z}+D_{4} \varepsilon_{\perp}\right) \\
E_{C}= & E_{C}(0)+\left(a_{c z}-D_{1}\right) \varepsilon_{z z}+\left(a_{c t}-D_{2}\right) \varepsilon_{\perp}
\end{aligned}
$$

Here $\varepsilon_{\perp}=\varepsilon_{x x}+\varepsilon_{y y}$ and $\varepsilon_{z z}$ are the strain components in and perpendicular to the $c$ plane. Note that the valence-band deformation potentials $D_{1}\left(D_{2}\right)$ are combined with the conduction-band deformation potentials $a_{c z}\left(a_{c t}\right)$. Uniaxial strain in the $c$ plane $\left(\varepsilon_{y y}=\varepsilon_{z z}=0\right.$, $\left.\varepsilon_{x x} \neq 0\right)$ changes the crystal symmetry from $C_{6 v}$ to $C_{2 v}$. This lifts the degeneracy of the $\Gamma_{6}$ state and leads to the following splitting between the $\mathrm{HH}$ and $\mathrm{LH}$ bands:

$$
\Delta E=\left|E_{H H}-E_{C H}\right|=2\left|D_{5}\left(\varepsilon_{x x}-\varepsilon_{y y}\right)\right|
$$

From the slopes of the transition energies under biaxial $\left(\varepsilon_{x x}=\varepsilon_{y y} \neq 0, \varepsilon_{z z}=0\right)$ or uniaxial strain $\left(\varepsilon_{x x}=\varepsilon_{y y}=\right.$ $\left.0, \varepsilon_{z z} \neq 0\right)$ ) the deformation potentials $a_{c z}-D_{1}, a_{c t}-D_{2}$, $D_{3}$ and $D_{4}$ can be obtained, whereas uniaxial strain in the $c$ plane yields $D_{5}$.

We calculate the dependence of the transition energies on strain by computing the band structure using HSE for three different strain conditions. The internal displacement parameter $u$ is fully relaxed in every case. Figure 1 shows the crystal-field splitting $\left(\Delta_{c r}\right)$ of GaN under biaxial strain in the $c$ plane for the strain range $\pm 3 \%$. One might have expected the dependence to be linear, but instead a clearly nonlinear behavior is observed, which can be well described by a quadratic dependence as demonstrated by the fitted curve. It implies that the slope (needed to determine the deformation potentials) differs for different lattice parameters. Panels (b) and (c) show that $D_{3}$ and $D_{4}$ vary by more than $40 \%$ in the strain range $\pm 3 \%$. Similar nonlinearities are observed for AlN and InN. For systems with large internal strain components, such as InGaN alloys grown on GaN substrates, these nonlinearities should be taken into account.

By constraining the strain to realistic strain conditions in the linear regime around the experimental lattice pa-

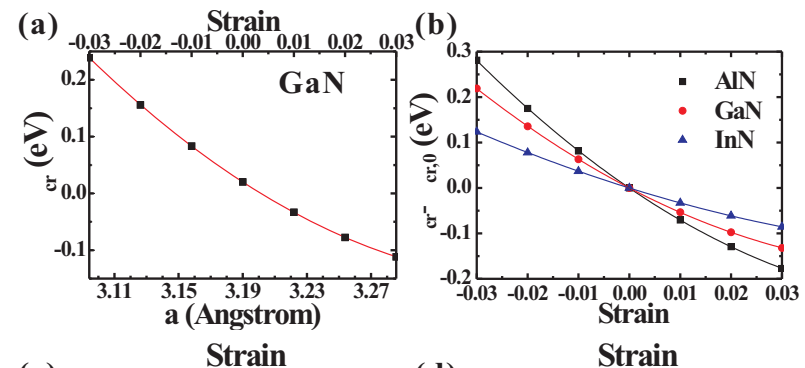

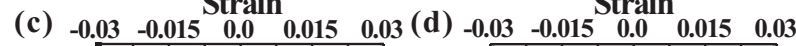

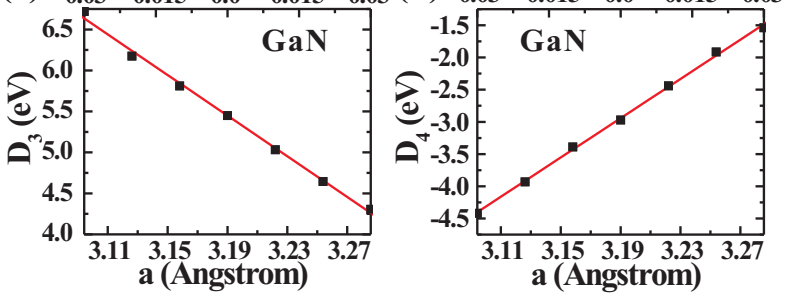

FIG. 1: (color online) (a) Calculated crystal-field splitting $\Delta_{c r}$ of wurtzite GaN under biaxial strain in the $c$ plane. (b) The energy difference of crystal-field splitting between strained $\left(\Delta_{c r}\right)$ and unstrained case $\left(\Delta_{c r, 0}\right)$ of wurzite AlN, $\mathrm{GaN}$, and InN under biaxial strain in the $c$ plane. The lower panels show the calculated deformation potentials $D_{3}$ (c) and $D_{4}$ (d) of GaN as a function of lattice parameter $a$. The fitted curves are quadratic in (a) and (b) and linear in (c) and (d).

TABLE II: Deformation potentials (eV) of wurtzite AlN, GaN

\begin{tabular}{|c|c|c|c|c|c|c|}
\hline & $\mathrm{AlN}$ & \multicolumn{4}{|c|}{ GaN } & InN \\
\hline & HSE & exp (range) & VM & HSE & $G_{0} W_{0}$ & HSE \\
\hline$\overline{a_{c z}-D_{1}}$ & -4.31 & $-9.6 \ldots-3.1$ & -4.90 & -5.81 & -5.33 & -3.62 \\
\hline$a_{c t}-D_{2}$ & -12.11 & $-11.8 \ldots-8.1$ & -11.30 & -8.92 & -8.84 & -4.60 \\
\hline$D_{3}$ & 9.12 & $1.4 \ldots 8.2$ & 8.20 & 5.47 & 5.80 & 2.68 \\
\hline$D_{4}$ & -3.79 & $-4.1 \ldots-0.7$ & -4.10 & -2.98 & -3.09 & -1.74 \\
\hline$D_{5}$ & -3.23 & $-4.7 \ldots-3.3$ & -4.60 & -2.82 & - & -2.07 \\
\hline
\end{tabular}
and InN. For GaN the range of experimentally determined deformation potentials and the recommended values by Vurgaftman and Meyer (VM) (Ref.13) are also shown.

rameters we derive a consistent and complete set of deformation potentials from the HSE calculations which are listed in Table II. With the exception of $a_{c z}-D_{1}$ the deformation potentials decrease in absolute value from AlN to InN. Due to the appreciable scatter in the experimentally determined deformation potentials for GaN we only report the range of measured values in Table II. Apart from $D_{5}$, which is slightly larger than the largest experimental value, the HSE values all fall within the experimental range. No experimental results are available for AlN or InN so far. Cross-checks for GaN show that the HSE values are in good agreement with those obtained from $G_{0} W_{0}$ calculations ${ }^{28}$ - currently the band structure method of choice for solids - which validates the suitability of the HSE method for studying the strain effects for group-III-nitrides. This also makes our set of deformation potentials consistent with the Luttinger parameters of Rinke et $a l .{ }^{26}$

Combining available theoretical and experimental data Vurgaftman and Meyer suggested a set of recommended deformation potentials for $\mathrm{AlN}, \mathrm{GaN}$ and $\mathrm{InN}$ in $2003 .{ }^{13}$ 
In Table II we list their values for GaN. With the exception of $a_{c z}-D_{1}$ our HSE values give smaller absolute values. Vurgaftman and Meyer's recommended values for AlN were taken from LDA calculations by Shimada et al. ${ }^{29}$ These values are in good agreement with our HSE calculations except that HSE gives an absolutely larger $a_{c z}-D_{1}$ value. This is consistent with our own LDA calculations and illustrates that the band-gap related deformation potentials (such as $a_{c z}-D_{1}$ ) are most sensitive to exchange-correlation and require a band-gap corrected approach, while the deformation potentials dependent only on valence-band energy differences $\left(D_{3}, D_{4}\right.$, and $\left.D_{5}\right)$ are less sensitive. ${ }^{30}$ In the absence of any computational or experimental values for InN, Vurgaftman and Meyer recommended to use the same deformation potentials as for GaN. Our HSE values show that this is not a good approximation, with differences in magnitude as large as several $\mathrm{eV}$.

Our first-principles calculations also allow us to assess the accuracy of the quasicubic approximation, which assumes that the similarity of local atomic coordination between wurtzite and zinc-blende structures leads to a correlation of the physical properties of the wurtzite structure with those of the zinc-blende structure along the $<111>$ direction. In the quasicubic approximation, the deformation potentials are related as follows: $D_{3}=-2 D_{4}, D_{1}+D_{3}=D_{2}$ and $D_{3}+4 D_{5}=\sqrt{2} D_{6}$. The quasicubic approximation is often made in experimental studies, because it eliminates the explicit dependence on three of the deformation potentials. As a test, we checked the value of $D_{3}+2 D_{4}$ (which should be zero in the quuasicubic approximation), finding $1.43 \mathrm{eV}$ for AlN, -0.52 for $\mathrm{GaN}$ and $-0.88 \mathrm{eV}$ for $\mathrm{InN}$. Omitting the anisotropy of the wurtzite phase by invoking the quasicubic approximation clearly introduces inaccuracies in the determination of deformation potentials.

In conclusion, the effects of strain on the band structure of wurtzite AlN, GaN, and InN have been studied using a first-principles approach based on hybrid-functional DFT. We observe nonlinearities in the strain dependence that should be taken into account in highly strained nitride materials and alloys. For the linear regime around the experimental lattice parameters, we present a complete and consistent set of deformation potentials. This set of parameters constitutes important input for nitride heterostructure device modeling and facilitates the prediction of band positions under realistic strain conditions.

This work was supported by the Solid State Lighting and Energy Center at the University of California, Santa Barbara. It made use of the CNSI Computing Facility (NSF grant No. CHE-0321368) and TeraGrid computing resources (NSF grant No. DMR070072N). P. R. acknowledges the support of the Deutsche Forschungsgemeinschaft. We thank A. Janotti for fruitful discussions and G. Kresse for providing an unreleased version of the VASP code.
1 S. Nakamura, Science 281, 956 (1998).

2 S. Nakamura, M. Senoh, S. Nagahama, N. Iwasa, T. Yamada, T. Matsushita, Y. Sugimoto, and H. Kiyoku, Appl. Phys. Lett. 69, 4056 (1996).

3 Y. Taniyasu, M. Kasu, and T. Makimoto, Nature 441, 325 (2006).

4 B. S. Kang, F. Ren, B. P. Gila, C. R. Abernathy, and S. J. Pearton, Appl. Phys. Lett. 84, 1123 (2004).

5 S. Kako, C. Santori, K. Hoshino, S. Götzinger, Y. Yamamoto, and Y. Arakawa, Nature Materials 5, 887 (2006).

${ }^{6}$ H. S. Jung, Y. J. Hong, Y. Li, J. Cho, Y. Kim, and G. Yi, ACS Nano 2, 637 (2008).

7 Y. C. Shen, G. O. Mueller, S. Watanabe, N. F. Gardner, A. Munkholm, and M. R. Kramers, Appl. Phys. Lett. 91, 141101 (2007).

8 K. T. Delaney, P. Rinke, and C. G. Van de Walle, Appl. Phys. Lett. 94, 191109 (2009).

9 G. D. Chen, M. Smith, J. Y. Lin, H. X. Jiang, S. H. Wei, M. A. Kahn, and C. J. Sun, Appl. Phys. Lett. 68, 2784 (1996).

10 B. Gil, O. Briot, and R. L. Aulombard, Phys. Rev. B 52, R17028 (1995).

11 W. Shan, A. J. Fischer, J. J. Song, G. E. Bulman, H. S. Kong, M. T. Leonard, W. G. Perry, M. D. Bremser, and R. F. Davis, Appl. Phys. Lett. 69, 740 (1996).

12 P. Misra, U. Behn, O. Brandt, H. T. Grahn, B. Imer, S. Nakamura, S. P. DenBaars, and J. S. Speck, Appl. Phys. Lett. 88, 161920 (2006).

13 I. Vurgaftman and J. R. Meyer, J. Appl. Phys. 94, 3675 (2003).

14 P. Perlin, L. Mattos, N. A. Shapiro, and J. Kruger, J. Appl. Phys. 85, 2385 (1999).

15 S. Chichibu, T. Azuhata, T. Sota, H. Amano, and
I. Akasaki, Appl. Phys. Lett. 70, 2085 (1997).

16 A. A. Yamaguchi, Y. Mochizuki, C. Sasaoka, A. Kimura, M. Nido, and A. Usui, Appl. Phys. Lett. 71, 374 (1997).

17 H. Y. Peng, M. D. McCluskey, Y. M. Gupta, M. Kneissl, and N. M. Johnson, Phys. Rev. B 71, 115207 (2005).

18 S. X. Li, J. Wu, E. E. Haller, W. Walukiewicz, W. Shan, H. Lu, and W. J. Schaff, Appl. Phys. Lett. 83, 4963 (2003).

19 S. L. Chuang and C. S. Chang, Phys. Rev. B 54, 2491 (1996).

20 M. Suzuki and T. Uenoyama, Jpn. J. Appl. Phys. 35, L953 (1996).

21 K. Kim, W. R. L. Lambrecht, and B. Segall, Phys. Rev. B 53, 16310 (1996).

22 J. Heyd, G. E. Scuseria, and M. Ernzerhof, J. Chem. Phys. 118, 8207 (2003).

23 J. Heyd, G. E. Scuseria, and M. Ernzerhof, J. Chem. Phys. 124, 219906 (2006).

${ }^{24}$ G. Kresse and J. Furthmüller, Phys. Rev. B 54, 11169 (1996).

25 J. P. Perdew, K. Burke, and M. Ernzerhof, Phys. Rev. Lett. 77, 3865 (1996).

${ }^{26}$ P. Rinke, M. Winkelnkemper, A. Qteish, D. Bimberg, J. Neugebauer, and M. Scheffler, Phys. Rev. B 77, 075202 (2008).

27 G. L. Bir and G. E. Pikus, Symmetry and Strain-Induced Effects in Semiconductors (Wiley, New York, 1974).

28 P. Rinke, A. Qteish, J. Neugebauer, C. Freysoldt, and M. Scheffler, New J. Phys. 7, 126 (2005).

29 K. Shimada, T. Sota, and K. Suzuki, J. Appl. Phys. 84, 4951 (2003).

${ }^{30}$ Q. Yan, P. Rinke, M. Scheffler, and C. G. Van de Walle, in preparation. 